\title{
Do non-targeted gastric biopsies affect the management of patients, and can a simple protocol and education reduce the rate?
}

\author{
Victoria Gibson, ${ }^{1}$ Stephanie Needham, ${ }^{2}$ Manu Nayer, ${ }^{1}$ Nick P Thompson ${ }^{1}$
}

'Department of

Gastroenterology, Newcastle upon Tyne Hospitals NHS Foundation Trust, Freeman Hospital, Newcastle upon Tyne, UK

${ }^{2}$ Department of Cellular Pathology, Newcastle upon Tyne Hospitals NHS Foundation Trust, Royal Victoria Infirmary, Newcastle upon Tyne, UK

\section{Correspondence to}

Dr Victoria Gibson, Department of Gastroenterology, Newcastle upon Tyne Hospitals NHS foundation trust, Freeman Hospital, Freeman Road, Newcastle upon Tyne, UK ; gibsonvm@doctors.org.uk

Received 17 January 2017 Revised 1 June 2017

Accepted 3 July 2017

Published Online First

10 August 2017

\section{CrossMark}

To cite: Gibson $V_{\text {, }}$

Needham S, Nayer M, et al.

Frontline Gastroenterology

2018;9:67-72.

\begin{abstract}
Objective To determine whether development of localised protocol could reduce the number of non-targeted gastric biopsies taken at endoscopy, without risking harm from nondetection of malignant conditions.
\end{abstract}

Design Retrospective analysis of patient records over a 3-month period in 2013, repeated in 2015 following intervention.

Setting Two UK teaching hospitals

Patients Patient record data on indication for endoscopy, endoscopy findings, histopathology results and patient outcome.

Interventions Guidance on upper gastrointestinal biopsy in the form of a new trust-wide protocol, as well as lecture-based education.

Main outcome measures Rates of non-targeted and targeted biopsies before and after intervention, and differences between grade of endoscopist.

Results Between 2013 and 2015, there was a $36 \%$ reduction in non-targeted biopsies $(10.4 \%$ vs $6.7 \%, p=0.001)$, predominantly within registrar and nurse endoscopist groups, with reduction in non-targeted biopsies of $9.5 \%$ and $64 \%$, respectively. Percentage of targeted biopsies remained relatively static, $7.9 \%$ and $8.2 \%$. In 2013, $92 \%$ of non-targeted biopsies had no management change based on histology; in 2015 this was $90 \%$. Of patients with alteration to management, only $0.4 \%$ and $0.7 \%$ were due to malignancy, in known high-risk patients. Reduction in non-targeted biopsies resulted in estimated annual savings in this trust of $£ 36,000$.

Conclusion Development of local protocol reduces the numbers of non-targeted biopsies taken, without risk of harm from nondetection of malignant conditions, enabling a significant reduction in workload within busy histopathology services, with significant cost savings. Localised protocols are adaptable to local population demographics.

\section{INTRODUCTION}

Gastric biopsy remains a critical adjunct in the detection and diagnosis of significant gastric pathology including malignancy, as well as enabling the exclusion of malignancy where endoscopy has shown suspicious features in the gastric mucosa. A system for biopsy must be cost-effective yet robust enough to ensure detection of gastric cancers. This includes patients with 'alarm' symptoms (eg, weight loss, advanced age), and 'high-risk' patients (eg, those with a strong family history where there should be a low threshold for biopsy). ${ }^{1}$

However, unnecessary biopsy sampling of the gastric mucosa for histological analysis is both costly, and does not alter the management of the majority of patients. ${ }^{2}$ Examples include where gastroscopy (OGD) demonstrates 'gastritis' in a patient without 'alarm' features, or the biopsy is taken solely for Helicobacter pylori $(\mathrm{Hp})$ detection in cases where another test would be as effective and less expensive. Taking non-targeted or unnecessary biopsies from gastric mucosa remains widespread practice. Research to date has shown that in the majority of cases there is no benefit to the patient in taking a gastric biopsy where there is no clear indication. ${ }^{3}$ Biopsy of normal or non-suspicious mucosa has not been shown to increase the finding of neoplasia. ${ }^{4}$

Histopathology resources are limited and under pressure. It is essential that we practice cost-effective yet diagnostically sensitive methods of obtaining biopsies during endoscopy.

Reasons for the high percentage of 'unnecessary' biopsy may be due to global and local lack of guidelines for necessary biopsy within the gastrointestinal tract. 
The Sydney System ${ }^{5}$ for biopsy remains the best known guidance; however, it may not adapt to local demands and does not provide specific guidance on what does not require biopsy. Increased sampling of non-targeted biopsies may also relate to the grade of endoscopist, as those with less experience may be more inclined to take unnecessary biopsies, being less confident to manage risk or make endoscopic diagnoses. ${ }^{6}$

Shepherd and Valori have suggested that the development of local guidelines for gastric biopsy may reduce the number of unnecessary biopsies, while allowing for local adjustments within practice. ${ }^{7}$

\section{METHODS}

Initial data were collected over a 3-month period between 2 January 2013 and 31 March 2013, across two teaching hospital sites within the same trust. Details of patients who had undergone gastric biopsy during this period were obtained from our histopathology database, enabling us to access histopathology results for each biopsy taken. We were then able to access our endoscopy database for each patient. Data were obtained on patient demographics, reason for endoscopy, endoscopy findings/appearance of gastric mucosa, grade of endoscopist and whether an ureasebased Helicobacter test (UBHT) was taken and its result. All endoscopists were either in training with an experienced trainer, or were Joint Advisory Group on GI Endoscopy (JAG) accredited to be independent.

By accessing the electronic patient medical record database, we were able to determine whether the biopsy taken was targeted or non-targeted, and whether the biopsy histopathology result altered the management of the patient. A targeted biopsy was defined as the presence at OGD of a polyp, ulcer or any other lesion or appearance at endoscopy documented by the operator as being suspicious or suspected malignancy. Non-targeted biopsy was any other appearance including normal, and 'gastritis'.

To look at cost-effectiveness, we obtained details on the cost of biopsy processing from our histopathology department. The cost of a biopsy set included manpower and processing costs within histopathology, and we also looked at the combined cost of UBHT plus endoscopic forceps use.

Following on from the initial results, education within the trust was provided by two means. The first was oral presentations on the findings of the first data collection period, along with a question and answer session to obtain suggestions for the second intervention. This was developing a local protocol with guidance on when to obtain a biopsy at OGD, for use by both medical and surgical departments. The protocol was presented as a simple flow chart which was placed within all endoscopy theatres across the two sites (figure 1).

Once education had taken place and protocol guidance had been in place for 7 months, comparative data were collected for patients who had undergone gastric biopsy over a further 3 -month period, between 1 June 2015 and 31 August 15. This was analysed in an identical fashion so the two periods could be compared. This comparison included looking at the differences between grades of endoscopist. We performed $\chi^{2}$ tests to assess the statistical significance of our data.

\section{RESULTS}

\section{Demographics}

Data for the first cycle of audit were collected from 2 January 2013 to 30 March 2013, and the second cycle from 1 June 2015 to 31 August 2015.

The age range for each cycle was similar; age 18-97 years in 2013 and age 18-93 years in 2015. Median age was 66 years for both.

In 2013, 419 biopsy sets were collected with a total of 2288 OGDs performed in this 3 -month period, giving a biopsy rate of $18.3 \%$. In 2015, 333 biopsy sets were collected with total OGDs in the 3 -month period of 2244 , giving a biopsy rate of $14.8 \%, p=0.0001$. Table 1 gives a breakdown of data collection by year and grade of endoscopist.

\section{Non-targeted biopsy data}

In 2013, 57\% of biopsy sets were non targeted $(n=239 / 419)$. This represented $10.4 \%$ of the total number of OGDs. In $2015,45 \%$ of the biopsy sets were non-targeted $(n=150 / 333)$; this represented $6.7 \%$ of the total number of OGDs, $\mathrm{p}=0.001$. Table 2 demonstrates the histopathology result of non-targeted biopsies by year.

In 2013, of the non-targeted biopsy sets, 92\% $(n=220)$ had no change to management based on histology. In 2015, 90\% ( $\mathrm{n}=135)$ had no change to management based on histology.

The 3\% unknown results in 2015 could not be obtained from computer histology records, but on reviewing patient records, none resulted in any change in management as a result of histology.

In 2013, 8\% $(n=19)$ had patient management changed based on histology:

One had a gastrectomy (histology showed 'high suspicion of adenocarcinoma' with 'normal' OGD appearance- - a man aged 42 years undergoing surveillance for strong family history of gastric adenocarcinoma.

Six had eradication therapy as no UBHT was documented as being taken at time of OGD.

Six had recommendation to GP for eradication therapy as unclear on OGD of UBHT result.

Two were referred for further $\mathrm{Hp}$ testing as histology showed 'active gastritis possibly related to $\mathrm{Hp}$ but no organisms', and no UBHT documented.

Three patients had letters to general practitioner (GP) recommending proton pump inhibitor therapy (PPI) as histology showed gastritis (but all showed gastritis at OGD).

One patient was recommended for repeat OGD in 1 year due to low-grade dysplasia on histology. 


\section{Biopsy protocol during upper $\mathrm{Gl}$ endoscopies: Newcastle Hospitals}

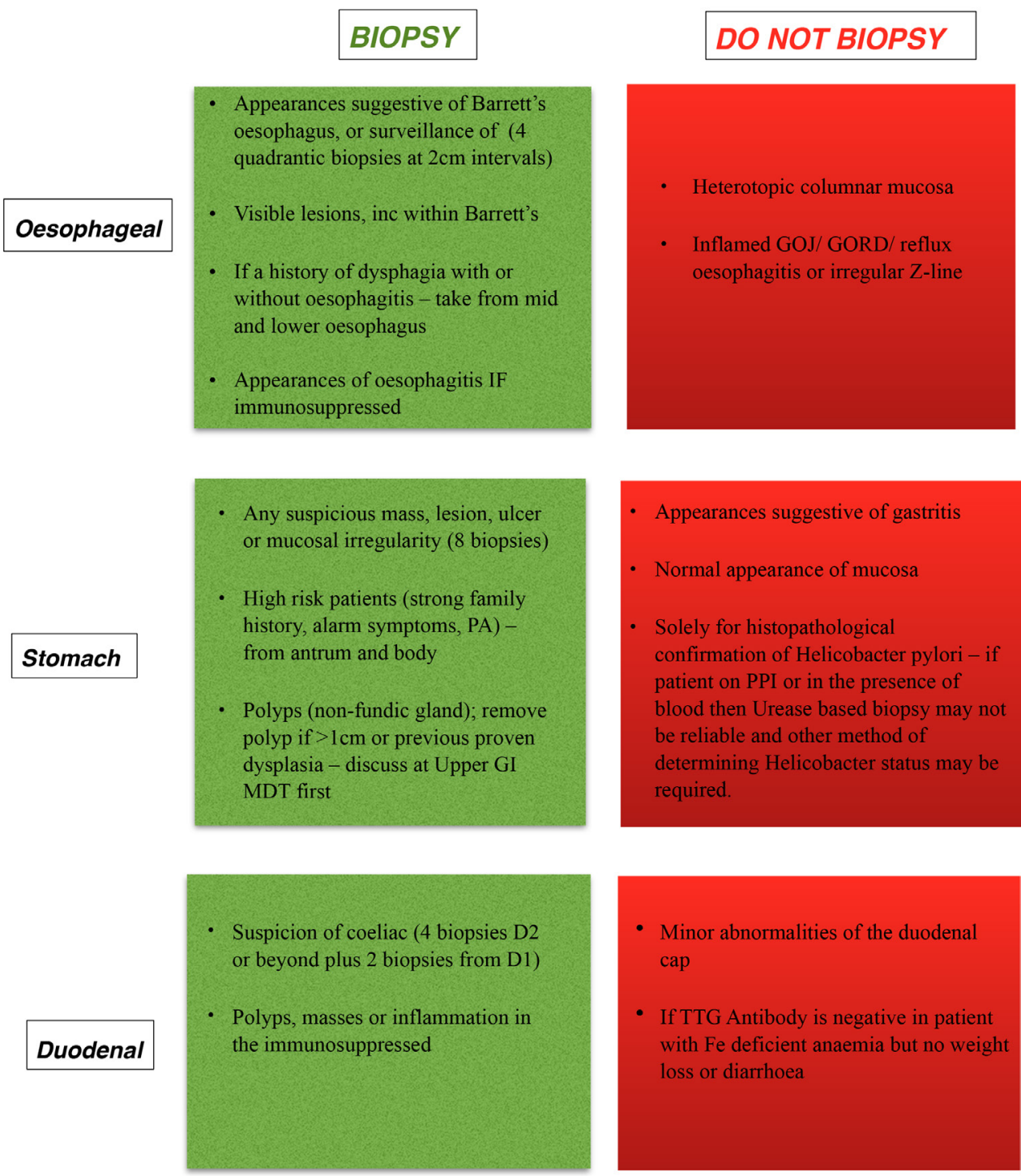

Figure 1 Newcastle hospitals protocol for biopsy sampling during upper gastrointestinal (GI) endoscopy.

In $2015,10 \%(n=15)$ had patient management changed based on histology:

One patient had $\mathrm{Hp}$-associated gastritis on histology, and microbiology subsequently confirmed Hp sensitivities (patient was under care for resistant $\mathrm{Hp})$.

One patient had lymphoma confirmed on biopsy (patient was under follow-up for lymphoma, and OGD showed nodular gastritis).

Seven patients were recommended for Hp treatment by their GP if not already received it as biopsies showed Hp, and UBHT was not documented as having been performed.

Six patients were recommended for $\mathrm{Hp}$ treatment by their GP if not already received it as biopsies showed $\mathrm{Hp}$, and UBHT was documented as being done but result was not recorded at time of OGD.

\section{DISCUSSION}

Gastric biopsy and histological assessment provide a critical adjunct to endoscopic assessment of the gastrointestinal tract. However, taking only appropriate biopsies can considerably reduce histopathological cost and workload in a health system under pressure. ${ }^{7}$ Several studies have supported the theory that there is no increased detection of malignancy by routine non-targeted biopsy. ${ }^{4}$ Despite this, there is limited general guidance. Guidance within Leeds Hospitals is one example of where such guidelines exist. ${ }^{7}$

Data were collected over a 3-month period during each audit cycle to allow comparison. This resulted in a very similar total number of endoscopies; however, the biopsy rate was significantly lower in the 2015 cycle. Demographics of the patient groups were similar in both cycles, enabling us to draw direct comparisons. 
Table 1 Breakdown of data collection by year and grade of endoscopist

\begin{tabular}{|c|c|c|c|}
\hline Year of data collection & 2013 & 2015 & \\
\hline Number of endoscopies & 2288 & 2244 & \\
\hline Number of biopsy sets (rate) & $419(18.3 \%)$ & $333(14.8 \%)$ & $19 \%$ reduction $p=0.0001$ \\
\hline Number of non-targeted biopsies (rate) & $239(10.4 \%)$ & $150(6.7 \%)$ & $36 \%$ reduction $p=0.001$ \\
\hline \multicolumn{4}{|l|}{ Consultant } \\
\hline Number of endoscopies & 949 & 897 & \\
\hline Number of biopsy sets (rate) & $90(9.5 \%)$ & $105(11.7 \%)$ & $19 \%$ increase \\
\hline Number of targeted biopsies (rate) & $45(4.7 \%)$ & $63(7 \%)$ & \\
\hline Number of non-targeted biopsies (rate) & $45(4.7 \%)$ & $42(4.7 \%)$ & $0 \%$ reduction $p=0.161$ \\
\hline \multicolumn{4}{|l|}{ Registrar } \\
\hline Number of endoscopies & 806 & 625 & \\
\hline Number of biopsy sets (rate) & $136(16.9 \%)$ & $100(16 \%)$ & $5 \%$ reduction \\
\hline Number of targeted biopsies (rate) & $76(9.4 \%)$ & $58(9.3 \%)$ & \\
\hline Number of non-targeted biopsies (rate) & $60(7.4 \%)$ & $42(6.7 \%)$ & $9.5 \%$ reduction $p=0.746$ \\
\hline \multicolumn{4}{|l|}{ Nurse endoscopist } \\
\hline Number of endoscopies & 533 & 722 & \\
\hline Number of biopsy sets (rate) & $187(35 \%)$ & $125(17.3 \%)$ & $51 \%$ reduction \\
\hline Number of targeted biopsies (rate) & $54(10 \%)$ & $59(8.2 \%)$ & \\
\hline Number of non-targeted biopsies (rate) & $133(25 \%)$ & $66(9 \%)$ & $64 \%$ reduction $p=0.001$ \\
\hline
\end{tabular}

This reduction in biopsy rate was only seen in non-targeted biopsy rate which reduced by $36 \%$ and was very likely to have been due to the development of a guidance document (figure 1), and education. Simultaneously, the percentage of targeted biopsies taken remained static.

Assuming a cost for each biopsy set as $£ 103.51$, this represents an annual saving for our trust of $£ 36432$. This assumes that biopsies are taken on each occasion for UBHT to diagnose the presence or not of Hp.

Both data sets showed that the vast majority of non-targeted biopsies did not alter the management of the patient. In the instances where this was the case, this largely centred around $\mathrm{Hp}$ treatment. The two patients who had serious abnormalities detected on histology following 'normal' endoscopy, were patients who would be deemed 'high-risk', requiring surveillance endoscopic biopsies.

\section{OPERATOR DEPENDENCY}

Both registrar and nurse endoscopist grades showed a reduction in the percentage of non-targeted biopsies taken, with the nurse endoscopist grade showing the largest reduction. This could be purported to

\begin{tabular}{ll}
\hline $\begin{array}{l}\text { Table } 2 \text { Histopathology result of non-targeted biopsies } \\
\text { by year }\end{array}$ \\
\hline $2013(n=239)$ & $2015(n=150)$ \\
\hline $72 \%$ gastritis $(n=173)$ & $70 \%$ gastritis $(n=105)$ \\
$16 \%$ Hp $(n=38)$ & $17 \%$ Hp $(n=26)$ \\
$11 \%$ normal $(n=26)$ & $9 \%$ normal $(n=14)$ \\
$0.5 \%$ low-grade dysplasia $(n=1)$ & $3 \%$ unknown $(n=4)$ \\
$0.5 \%$ adenocarcinoma $(n=1)$ & $0.7 \%$ lymphoma $(n=1)$ \\
\hline
\end{tabular}

p. Helicobacter pylori. be secondary to education provision and especially the development of a publicised protocol. Consultants already had a low rate of non-targeted biopsies and therefore the differential reduction is smaller. Within this low rate, there may have been something particular about those patients precipitating the decision to biopsy, not explicit in the endoscopy report.

Number of biopsies taken at OGD may vary between operator, often with no additional detection of pathology, ${ }^{6}$ which may be attributed to balancing and accepting risk. Development of specific guidance is therefore particularly helpful.

There are also recent data suggestions that missed cancers may be more to do with poor visual detection of abnormality at endoscopy than to do with non-biopsy of areas that look normal. ${ }^{8}$ This includes length of time taken to perform endoscopy. ${ }^{9}$ Scrutinising how we perform endoscopies to enable optimal visualisation of the gastric mucosa may improve this.

\section{HELICOBACTER DETECTION}

The vast majority of alterations to patient management were based around $\mathrm{Hp}$ treatment. In a high percentage of these, it was unclear from documentation whether the patient had already been treated; therefore, the actual percentage of patients who experienced an alteration to their management may actually be lower than we reported in both years. Taking histology for the sole purpose of Helicobacter testing is expensive. There are cheaper and reliable alternatives to Helicobacter testing other than histology based, ${ }^{10}$ including the UBHT, and these should be used instead where possible. ${ }^{11}$ 


\section{LIMITATIONS}

Histology showing intestinal metaplasia was not categorised as changing the management of the patient in this study. Our data showed that this finding at histology did not change the management, although there have been recent data suggesting intestinal metaplasia is a precursor to malignancy and should be under surveillance. However, at present, even though intestinal metaplasia is commonly found in practice, there is no clear consensus on recommendations for surveillance to guide physicians. ${ }^{12-14}$ The European Society of Gastrointestinal Endoscopy Quality Improvement Initiative ${ }^{15}$ suggests taking four non-targeted biopsies to look for intestinal metaplasia or dysplasia as precursors following management of precancerous conditions and lesions in the stomach (MAPS)guidance. However, the performance measure excludes 'UGI endoscopy with normal gastric findings', and the denominator is 'all endoscopic examinations where assessment of the gastric cancer risk is considered clinically relevant'. We suggest patients who are deemed clinically to be 'at risk' of gastric cancer have biopsies taken as recommended, and therefore remains in line with the European QI recommendation. This also applies to patients with any suspicious mucosa at endoscopy, as in our study these patients were classed as having 'targeted' lesions and therefore would warrant biopsy.

Although ideally the same endoscopists would be reaudited, this was not practical as Registrars rotate on an annual or 6 monthly basis, and there is inevitable movement of staff.

Follow-up of patients beyond the end date of the study has not been performed to determine whether future

\section{Significance of this study}

\section{What is already known on this topic?}

- Unnecessary biopsy sampling of gastric mucosa does not alter management in the majority of patients, and is costly.

- Research to date has not shown an increase in the detection of neoplasia from obtaining non-targeted biopsies.

- However, it remains widespread practice and as a result, histopathology departments are often under significant workload pressures.

\section{What are the new findings?}

- Development of a local protocol reduces the numbers of non-targeted biopsies taken, without risk of harm from non-detection of malignant conditions.

- How might it impact on clinical practice in the foreseeable future?

- Localised protocols could be adapted to local population demographics, to enable a significant reduction in workload within histopathology services, with significant cost savings. malignant lesions developed; this would need to be done on a prospective basis in a long-term study.

Our data collection was restricted to those patients undergoing biopsy in a specific trust within the NorthEast of England. While the data collected were of sufficient number, the results may not be applicable to other parts of the world, due to varying incidences of gastric malignancy in different populations.

\section{CONCLUSIONS}

Development of a local protocol reduces the numbers of non-targeted biopsies taken, without the risk of harm to the patient from not detecting malignant conditions. This enables a significant reduction in workload within histopathology services, with significant cost savings. Localised protocols would allow adaptation to different population demographics.

Contributors The planning and reporting of this paper was contributed to by all named authors. Data collection was undertaken by VG (lead author), who also acts as guarantor for this paper.

Competing interests None declared.

Provenance and peer review Not commissioned; externally peer reviewed.

(C) Article author(s) (or their employer(s) unless otherwise stated in the text of the article) 2018. All rights reserved. No commercial use is permitted unless otherwise expressly granted.

\section{REFERENCES}

1 Yalamarthi S, Witherspoon P, McCole D, et al. Missed diagnoses in patients with upper gastrointestinal cancers. Endoscopy 2004;36:874-9.

2 Teriaky A, AlNasser A, McLean C, et al. The utility of endoscopic biopsies in patients with normal Upper Endoscopy. Can J Gastroenterol Hepatol 2016;2016:1-7.

3 Howat A, Boyd K, et al. Histopathology and cytopathology of limited or no clinical value. 2nd. Royal College of Pathologists, 2005.

4 Cadman B, Dixon MF, Wyatt JI. Value of routine, non-targeted biopsies in the diagnosis of gastric neoplasia. J Clin Pathol 1997;50:832-4.

5 Dixon MF, Genta RM, Yardley JH, et al. Classification and grading of gastritis. the updated Sydney System. International Workshop on the Histopathology of Gastritis, Houston 1994. Am J Surg Pathol 1996;20:1161-81.

6 Williams J, Russell I, Durai D, et al. What are the clinical outcome and cost-effectiveness of endoscopy undertaken by nurses when compared with doctors? A Multi-Institution nurse Endoscopy Trial (MINuET). Health Technol Assess 2006;10:1195. iii-iv, ix-x.

7 Shepherd NA, Valori RM. The effective use of gastrointestinal histopathology: guidance for endoscopic biopsy in the gastrointestinal tract: table?1. Frontline Gastroenterol 2014;5:84-7.

8 Ang TL, Khor CJ, Gotoda T. Diagnosis and endoscopic resection of early gastric Cancer. Singapore Med J 2010;51:93-100.

9 Clin Gastroenterol Hepatol 2015;13:480-7.

10 Vaira D, Vakil N, Blood VD. Blood, urine, stool, breath, money, and Helicobacter pylori. Gut 2001;48:287-9.

11 Elwyn G, Taubert M, Davies S, et al. Which test is best for Helicobacter pylori? A cost-effectiveness model using decision analysis. Br J Gen Pract 2007;57:401-3.

12 Ballester V, Cruz-Correa M. Endoscopic surveillance of gastrointestinal premalignant lesions: current knowledge 


\section{ENDOSCOPY}

and future directions. Curr Opin Gastroenterol

2014;30:477-83.

13 Whiting JL, Sigurdsson A, Rowlands DC, et al. The long term results of endoscopic surveillance of premalignant gastric lesions. Gut 2002;50:378-81.

14 O'Connor A, McNamara D, O'Moráin CA. Surveillance of gastric intestinal metaplasia for the prevention of gastric
Cancer. Cochrane Database Syst Rev 2013:CD009322. Art. No.: CD009322.

15 Bisschops R, Areia M, Coron E, et al. Performance measures for upper gastrointestinal endoscopy: a european Society of gastrointestinal endoscopy quality improvement initiative. United European Gastroenterol J 2016;4:629-56. 